\title{
Parametric modal analysis of the brain-CSF-skull system
}

\section{Influence of the fluid-structure interaction}

\author{
Adil El Baroudi - Fulgence Razafimahery \\ Lalaonirina R. Rakotomanana
}

IRMAR (UMR CNRS 6625)

263 Avenue Général Leclerc

Campus de Beaulieu

F-35042 Rennes cedex

\begin{abstract}
Dynamical behavior of the head during an impact is important for analyzing the induced local damage or diffuse damage in the brain tissue. We determine in the present study the natural frequencies and the modal shapes of the system of brain, cerebro-spinal fluid and skull. Two models are presented in this work: an elastic-acoustic model assuming a rigid skull and an elastic-acoustic-elastic model assuming a deformable skull. It is shown that natural frequencies and more significantly the modal shapes are strongly influenced by the interaction between solid phases (brain and skull) and the cerebro-spinal fluid.

RÉSUMÉ. Le comportement dynamique de la tête lors d'un impact est de prime importance pour analyser les conséquences dans les lésions locales ou diffuses du cerveau. Nous déterminons dans ce travail les fréquences et modes propres d'un système formé par le cerveau, le liquide céphalo-rachidien et le crâne. La première partie de l'étude est consacrée à un modèle où le crâne est supposé rigide (modèle élastique-acoustique). La seconde partie présente un modèle dont le crâne est déformable. L'étude a montré que les fréquences propres et surtout les modes propres de vibrations sont très influencés par l'interaction entre les phases solides, le cerveau et le crâne et le fluide céphalo-rachidien.

KEYWORDS: modal analysis, cerebro-spinal fluid, fluid-structure interaction.

MOTS-CLÉS : analyse modale, liquide céphalo-rachidien, interaction fluide-structure.
\end{abstract}

DOI:10.3166/EJCM.18.55-66 @ 2009 Lavoisier, Paris

EJCM - 18/2009. Numerical models in biomechanics, pages 55 to 66 


\section{Introduction}

On the one hand, brain injuries constitute one of the major cause of death in road accidents. To understand how the brain gets injured during an accident, the mechanical response of the contents of the head during an impact has to be known. The consequences of the skull's vibrations are still poorly understood. It is probable that the low-frequency skull vibrations (below $200 \mathrm{~Hz}$ ) mainly cause deep cerebral lesions, while higher frequency vibrations have more consequences on the superficial cerebral structures e.g. (Willinger et al., 1996). Due to the presence of a thin layer of the cerebro-spinal fluid (CSF) between the brain and the skull, relative motion can occur and may explain many types of brain injury. First, intracerebral hematomas may appear due to bridging veins rupture. Then, contusions or bruising is likely to occur when the brain hits against the inner surface of the skull. Last, coup and contre-coup injuries can happen when the head is suddenly accelerated. The coup injury is caused by the brain hitting the interior of the skull; the contre-coup injury occurs directly opposite the blow due to a process called cavitation. One of the difficulties to apprehend the trauma brain injury (TBI) is the lack of informations about the stress distribution within the brain, the CSF and the skull because of the presence of a fluid phase. Solving fluid-structure mechanical interaction is thus expected to further improving our understanding of the impact of the head during an accident.

On the other hand, the knowledge of the flow dynamics and oscillations of the CSF in the intra-cranial space plays an important role for various human pathology in the everyday activities. Magnetic Resonance Imaging allows to capture pulsation of CSF in vivo (Kao et al., 2007), showing that main pulsations are respiratory modulated. More recent studies on the brain tend more and more to point out the role of wave within the brain tissue e.g. (Will et al., 2007). They experimentally showed that synchronization responses of brainwaves to periodic auditory stimuli had three components corresponding to different spectra: low frequencies $[1-5 \mathrm{~Hz}]$, intermediate frequencies $[3-8 \mathrm{~Hz}]$, and higher frequencies $[11-44 \mathrm{~Hz}]$. In addition, mechanical vibrations also play important role during measurement as Diffusion Tensor Imaging by means of Magnetic Resonance Imaging. Recent studies showed that the mechanical vibrations cannot be ignored and they should be considered when choosing the sequence parameters for Diffusion Tensor Imaging e.g. (Hiltunen et al., 2006). All these observations suggest us to better determine the natural frequencies and modal shapes of the brain, the CSF and the skull. Analytical methods were conducted to determine the frequency spectrum of the head-neck system e.g. (Charalambopoulos et al., 1997). Finite element method has been intensively used to model the head impact e.g. (Kleiven, 2002). Most of them neglected the mechanical role of the CSF. A basic question remain : what would be the role of the cerebro-spinal fluid for free and forced vibrations of the brain-CSF-skull system at low frequencies $\leq 200 \mathrm{~Hz}$ ? Numerical aspects of fluid-structure problems have been studied in the past e.g. (Morand et al., 1979). Most classical methods use the modal shapes of the solid phase for deriving the dynamical behavior of the whole (solid and fluid domains). In a general manner, classical methods may be sufficient if we are only interested in searching for 
the natural frequencies. However, these methods do not permit to obtain the modal shapes of the whole system which is essential for applying the modal projection when facing dynamical situation (forced vibrations or shocks).

The goal of the present work was to search for analytical and numerical modal shapes of the interacting brain-CSF-skull assuming elastic behavior for the brain and the skull and an acoustic wave propagation within the CSF. The model is designed to directly solve the coupled solid-fluid problem, conversely to classical method that only uses the modal shapes of the solid phase. The influence of the CSF compressibility and thickness on the natural frequencies and modal shapes was investigated. Last but not least, the use of simple models was deemed necessary since validation of the fluid-structure problems still remains a challenge.

\section{Basic models}

First, we develop linear plane-strain models and assume an inertial coupling, meaning that the longitudinal wave velocity is very large compared with the characteristic velocity of the fluid. The Helmholtz decomposition e.g. (Rakotomanana, To appear 2008) of the displacement field $\mathbf{u}_{i}=\nabla \varphi_{i}+\operatorname{rot}\left(\psi_{i} \mathbf{z}\right)$ and the acoustic assumption for CSF induce:

$$
\Delta \varphi_{i}+\frac{\omega^{2}}{c_{i L}^{2}} \varphi_{i}=0, \quad \Delta \psi_{i}+\frac{\omega^{2}}{c_{i T}^{2}} \psi_{i}=0, \quad \Delta p+\frac{\omega^{2}}{c_{0}^{2}} p=0
$$

where $c_{i L}, c_{i T}$ are the longitudinal and transversal wave velocities within the solid phase (brain : $\mathrm{i}=1$; skull : $\mathrm{i}=2$ ), and $c_{0}$ the sound velocity in the CSF respectively. The pressure is $p(\mathbf{x}, t)$ within the CSF and the stresses within solid phases are:

$$
\sigma\left(\mathbf{u}_{i}\right)=\lambda_{i}\left(\operatorname{div} \mathbf{u}_{i}\right) \mathbf{I}+2 \mu_{i} \varepsilon\left(\mathbf{u}_{i}\right)
$$

The two basic models developed in the present study are represented in Figure 1. The model is elastic-acoustic when the outer skull is rigid and elastic-acoustic-elastic when the skull is elastic. During a previous study, an experimental jig was developed for a three-dimensional analysis of a head during an impact (Hault-Dubrulle, 2007). Geometry and material properties of the present study were derived from this previous study (cf. Table 1).

Table 1. Geometry and material properties

\begin{tabular}{|c|c|c|c|c|c|}
\hline & $E[P a]$ & $\nu$ & $\rho\left[k g / \mathrm{m}^{3}\right]$ & $R[\mathrm{~m}]$ & $c_{0}[\mathrm{~m} / \mathrm{s}]$ \\
\hline$\Omega$ & & & 1000 & $R_{2}=0.090$ & 1450 \\
\hline$\Omega_{1}$ & $6,775.10^{5}$ & 0,48 & 1150 & $R_{1}=0.075$ & \\
\hline$\Omega_{2}$ & $7.10^{10}$ & 0,33 & 2750 & $R_{2}=0.095$ & \\
\hline
\end{tabular}




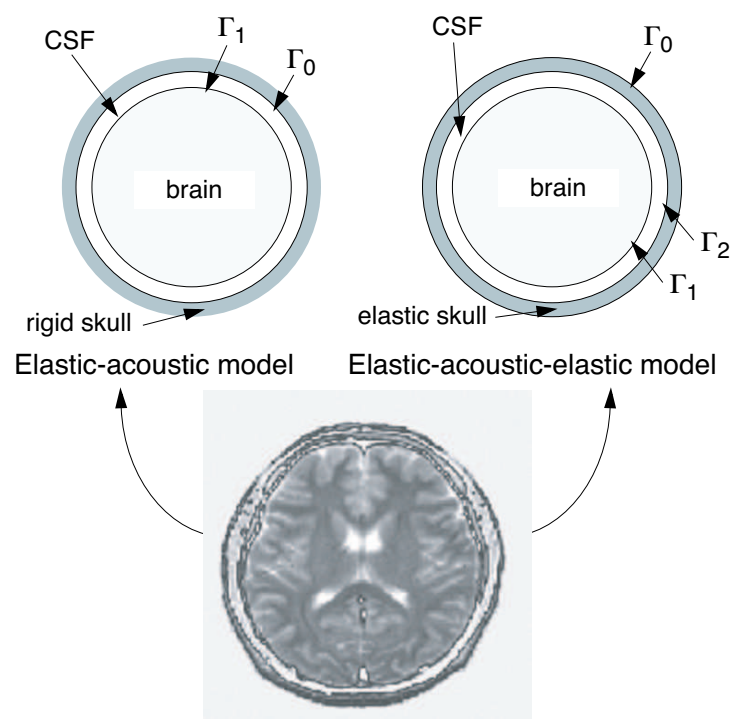

Figure 1. Elastic-acoustic and Elastic-acoustic-elastic models: brain $\left(\Omega_{1}\right)$, cerebrospinal fluid $(\Omega)$ and skull $\left(\Omega_{2}\right)$. Top view of a human brain-CSF-skull system

These values take into account the fluid rotational movement and then differ from those of (Charalambopoulos et al., 1997) which considered the brain tissue as an inviscid irrotational fluid. Analytical solutions of [1] take the form of :

$$
\begin{aligned}
\varphi_{1} & =A_{1} J_{n}\left(\frac{\omega r}{c_{1 L}}\right) \cos (n \theta), \quad \psi_{1}=B_{1} J_{n}\left(\frac{\omega r}{c_{1 L}}\right) \sin (n \theta) \\
p & =\left[A J_{n}\left(\frac{\omega r}{c_{0}}\right)+B Y_{n}\left(\frac{\omega r}{c_{0}}\right)\right] \cos (n \theta) \\
\varphi_{2} & =\left[A_{2} J_{n}\left(\frac{\omega r}{c_{2 L}}\right)+B_{2} Y_{n}\left(\frac{\omega r}{c_{2 L}}\right)\right] \cos (n \theta) \\
\psi_{2} & =\left[C_{2} J_{n}\left(\frac{\omega r}{c_{2 T}}\right)+D_{2} Y_{n}\left(\frac{\omega r}{c_{2 T}}\right)\right] \sin (n \theta)
\end{aligned}
$$

where $A, B, C$, and $D$ are constants. $J_{n}$ and $Y_{n}$ are the Bessel functions of the first and second kind respectively. Classical technique for analytical solving is based on the expression of the displacement and stress with these potentials and then their introduction in the boundary and interfacial conditions. 


\section{Rigid skull : Elastic-acoustic model}

We first consider the elastic-acoustic model where the skull is assumed rigid. The fluid-structure problem is governed by equations [1] with boundary conditions:

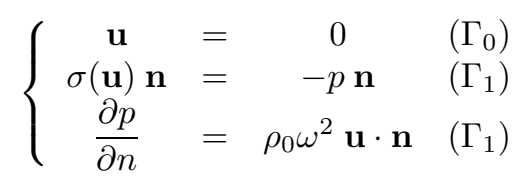

The last equation on $\left(\Gamma_{1}\right)$ is called transpiration condition. The transpiration condition expresses the dynamic compatibility of the two continua, or equivalently the projection of the linear momentum conservation, at the interface along the normal direction $\mathbf{n}$ of $\left(\Gamma_{1}\right)$. Analytical solutions: Solutions of elastic-acoustic problem [1] without the skull $i=2$ - are treated as follows. We write first the displacement and stress in terms of $\varphi$ and $\psi$ Then, the boundary conditions (2), say $\sigma_{r \theta}\left(R_{1}, \theta\right)=0$ and $\sigma_{r r}\left(R_{1}, \theta\right)=-p\left(R_{1}\right)$ allow to eliminate constants. Second, the transpiration condition at the interface $\left(\Gamma_{1}\right)$ implies the dispersion equation. Indeed, introducing the time dependence $\exp (j \omega t)$, natural frequencies of the problem [1] with conditions [2] satisfy the dispersion equation (El-Baroudi, 2007) :

$$
H_{e a}(\omega, n)=0
$$

Modal shapes are for radial displacement $X_{n m}(r, \theta)=U_{n m}(r) \cos (n \theta)$, azmuthal displacement $Y_{n m}=V_{n m}(r) \cos (n \theta)$, and pressure $p_{n m}(r, \theta)=K_{n m}(r) \cos (n \theta)$. Maple was used for extracting the natural frequencies $\left\{f_{e}(n, m)\right\}$ from Equation [3]. Numerical solutions: To this end, the variational formulation of the problem [1] with the conditions [2] holds:

$$
\left\{\begin{aligned}
\int_{\Omega_{1}} \sigma\left(\mathbf{u}_{1}\right): \varepsilon\left(\mathbf{v}_{1}\right) d v-\omega^{2} \int_{\Omega_{1}} \rho_{1} \mathbf{u}_{1} \cdot \mathbf{v}_{1} d v-\int_{\Gamma_{1}} p \mathbf{v}_{1} \cdot \mathbf{n} d \Gamma & =0 \\
\int_{\Omega_{2}} \nabla p \cdot \nabla \phi d v-\omega^{2}\left(\int_{\Omega_{2}} \frac{p \phi}{c_{0}^{2}} d v+\int_{\Gamma_{1}} \mathbf{u}_{1} \cdot \mathbf{n} \phi d \Gamma\right) & =0
\end{aligned}\right.
$$

$\forall\left(\mathbf{v}_{1}, \phi\right) \in V \times Q$ where $V=\left\{\mathbf{v}_{1} \in H^{1}\left(\Omega_{1}\right), \mathbf{v}_{1}=0\left(\Gamma_{0}\right)\right\}$ et $Q=H^{1}(\Omega)$. We used Lagrange elements in which $\mathbf{u}_{h} \in P_{2} \times P_{2}$ and $p_{h} \in P_{1}$, the discretization of the variational form lead to the non symmetrical system :

$$
\left[\begin{array}{cc}
K_{1} & -B_{1} \\
O & K_{p}
\end{array}\right]\left[\begin{array}{c}
U_{1} \\
P
\end{array}\right]-\omega^{2}\left[\begin{array}{cc}
M_{1} & O \\
M_{1 a} & M_{p}
\end{array}\right]\left[\begin{array}{c}
U_{1} \\
P
\end{array}\right]=\left[\begin{array}{l}
0 \\
0
\end{array}\right]
$$

Frequencies $f_{h}$ and modal shapes were obtained using the finite element method (Comsol software). First natural frequencies $f_{i}=\omega_{i} / 2 \pi$ are reported in Table 2.

Influence of the CSF compressibility: We investigated the influence of the CSF compressibility by varying the sound velocity $c_{0}[\mathrm{~m} / \mathrm{s}]$. For illustration, six first natural frequencies are reported in Table 3. Frequencies are independent of the fluid 
60 EJCM - 18/2009. Numerical models in biomechanics

Table 2. Natural frequencies and modal shapes

\begin{tabular}{|c|c|c|c|c|c|c|}
\hline & $f_{1}$ & $f_{2}$ & $f_{3}$ & $f_{4}$ & $f_{5}$ & $f_{6}$ \\
\hline$f_{e}$ & 38.67 & 69.88 & 90.69 & 100.51 & 130.34 & 138.85 \\
\hline$(\mathrm{n}, \mathrm{m})$ & $(2,1)$ & $(3,1)$ & $(1,1)$ & $(4,1)$ & $(5,1)$ & $(2,2)$ \\
\hline$f_{h}$ & 38.67 & 69.88 & 90.69 & 100.51 & 130.34 & 138.85 \\
\hline & $f_{7}$ & $f_{8}$ & $f_{9}$ & $f_{10}$ & $f_{11}$ & $f_{12}$ \\
\hline$f_{e}$ & 153.74 & 159.36 & 182.97 & 187.66 & 200.42 & 215.39 \\
\hline$(\mathrm{n}, \mathrm{m})$ & $(0,1)$ & $(6,1)$ & $(3,2)$ & $(7,1)$ & $(1,2)$ & $(8,1)$ \\
\hline$f_{h}$ & 153.74 & 159.36 & 182.97 & 187.66 & 200.42 & 215.39 \\
\hline
\end{tabular}

Table 3. Natural frequencies vs. CSF compressibility

\begin{tabular}{|c|c|c|c|c|c|c|}
\hline & $f_{1}$ & $f_{2}$ & $f_{3}$ & $f_{4}$ & $f_{5}$ & $f_{6}$ \\
\hline$c_{0}=10^{3} \mathrm{~m} / \mathrm{s}$ & 38.67 & 69.88 & 90.69 & 100.51 & 130.34 & 138.85 \\
\hline$c_{0}=500 \mathrm{~m} / \mathrm{s}$ & 38.66 & 69.87 & 90.68 & 100.47 & 130.29 & 138.85 \\
\hline$c_{0}=300 \mathrm{~m} / \mathrm{s}$ & 38.65 & 69.85 & 90.68 & 100.47 & 130.29 & 138.84 \\
\hline
\end{tabular}

compressibility. Therefore, the incompressibility assumption seems validated with the model. This means that $\omega^{2} / c_{0}^{2} \simeq 0$ and we can write $\Delta p=0$.

Influence of the CSF thickness: By varying the CSF thickness $e=R_{2}-R_{1}$, we found that natural frequencies augment with the CSF thickness (Table 4 ).

Table 4. Natural frequencies vs. CSF thickness

\begin{tabular}{|c|c|c|c|c|c|c|}
\hline & $f_{1}$ & $f_{2}$ & $f_{3}$ & $f_{4}$ & $f_{5}$ & $f_{6}$ \\
\hline$e=0.012 m$ & 34.24 & 62.94 & 87.19 & 91.82 & 120.42 & 133.35 \\
\hline$e=0.009 m$ & 29.42 & 55.07 & 81.64 & 83.96 & 108.57 & 128.23 \\
\hline$e=0.006 m$ & 23.87 & 45.57 & 68.82 & 80.96 & 93.07 & 117.89 \\
\hline$e=0.003 m$ & 16.80 & 32.75 & 50.51 & 69.70 & 78.17 & 89.99 \\
\hline
\end{tabular}

\section{Elastic-acoustic-elastic model : fixed skull}

Consider elastic skull, $\Gamma_{0}$ fixed. The problem is governed by [1] with conditions :

$$
\left\{\begin{array}{ccccc}
\sigma\left(\mathbf{u}_{i}\right) \mathbf{n} & = & -p \mathbf{n} & \left(\Gamma_{i}\right) & \\
\frac{\partial p}{\partial n} & = & \rho_{0} \omega^{2} \mathbf{u}_{i} \cdot \mathbf{n} & \left(\Gamma_{i}\right) & i=1,2 \\
\mathbf{u}_{2} & = & 0 & \left(\Gamma_{0}\right) &
\end{array}\right.
$$


Analytical solutions: Conditions on $\left(\Gamma_{1}\right)$ first simplify the pressure formulation. Then the clamping condition on $\Gamma_{3}$ allows to express the displacement $u^{2}=\left(u_{r}^{2}, u_{\theta}^{2}\right)$. Condition $\sigma_{r \theta}^{2}\left(R_{2}, \theta\right)=0$ gives new expressions of $u^{2}=\left(u_{r}^{2}, u_{\theta}^{2}\right)$ and $\left(\sigma_{r r}^{2}, \sigma_{r \theta}^{2}\right)$. Finally, the two boundary conditions at $\left(\Gamma_{2}\right)$ give the equation of dispersion. Natural frequencies of [1] with conditions [4] are thus solutions of the dispersion equation (El-Baroudi, 2007):

$$
H_{e a e_{-} r}(\omega, n)=0
$$

Modal shapes are for radial $X_{n m}(r, \theta)=U_{n m}(r) \cos (n \theta)$, for azimuthal $Y_{n m}=$ $V_{n m}(r) \cos (n \theta)$ displacements, and for pressure $p_{n m}(r, \theta)=K_{n m}(r) \cos (n \theta) . \mathrm{Nu}$ merical solutions: Finite element method is used to extract the natural frequencies and modal shapes. The variational form of [1] with conditions [4] holds:

$$
\left\{\begin{array}{c}
\int_{\Omega_{1}} \sigma\left(\mathbf{u}_{1}\right): \varepsilon\left(\mathbf{v}_{1}\right) d v-\omega^{2} \int_{\Omega_{1}} \rho_{1} \mathbf{u}_{1} \cdot \mathbf{v}_{1} d v-\int_{\Gamma_{1}} p \mathbf{v}_{1} \cdot \mathbf{n} d \Gamma=0 \\
\int_{\Omega_{2}} \sigma\left(\mathbf{u}_{2}\right): \varepsilon\left(\mathbf{v}_{2}\right) d v-\omega^{2} \int_{\Omega_{2}} \rho_{2} \mathbf{u}_{2} \cdot \mathbf{v}_{2} d v-\int_{\Gamma_{2}} p \mathbf{v}_{2} \cdot \mathbf{n} d \Gamma=0 \\
\int_{\Omega_{2}} \nabla p \cdot \nabla \phi d v-\omega^{2}\left(\int_{\Omega_{2}} \frac{p \phi}{c_{0}^{2}} d v+\sum_{i=1}^{2} \int_{\Gamma_{i}} \mathbf{u}_{i} \cdot \mathbf{n} \phi d \Gamma\right)=0
\end{array}\right.
$$

$\forall(\mathbf{v}, \phi) \in V \times Q$ in which $V=\left\{\left(\mathbf{v}_{1}, \mathbf{v}_{2}\right) \in H^{1}\left(\Omega_{1}\right) \times H^{1}\left(\Omega_{2}\right), \mathbf{v}_{2}=0\left(\Gamma_{0}\right)\right\}$ and $Q=H^{1}(\Omega)$. By using again Lagrange elements, where $\mathbf{u}_{h} \in P_{2} \times P_{2}$ and $p_{h} \in P_{1}$, discretization of the variational form induces a non symmetrical system:

$$
\left[\begin{array}{ccc}
K_{1} & O & -B_{1} \\
O & K_{2} & -B_{2} \\
O & O & K_{p}
\end{array}\right]\left[\begin{array}{c}
U_{1} \\
U_{2} \\
P
\end{array}\right]-\omega^{2}\left[\begin{array}{ccc}
M_{1} & O & O \\
O & M_{2} & O \\
M_{1 a} & M_{2 a} & M_{p}
\end{array}\right]\left[\begin{array}{c}
U_{1} \\
U_{2} \\
P
\end{array}\right]=\left[\begin{array}{l}
0 \\
0 \\
0
\end{array}\right]
$$

Maple and Comsol Multiphysics softwares are used to extract the first 12 natural frequencies $f_{i}=\omega_{i} / 2 \pi$.

Table 5. Natural frequencies : analytical vs. numerical

\begin{tabular}{|c|c|c|c|c|c|c|}
\hline & $f_{1}$ & $f_{2}$ & $f_{3}$ & $f_{4}$ & $f_{5}$ & $f_{6}$ \\
\hline$f_{e}$ & 38.67 & 69.88 & 90.69 & 100.51 & 130.34 & 138.85 \\
\hline$(\mathrm{n}, \mathrm{m})$ & $(2,1)$ & $(3,1)$ & $(1,1)$ & $(4,1)$ & $(5,1)$ & $(2,2)$ \\
\hline$f_{h}$ & 38.67 & 69.88 & 90.69 & 100.51 & 130.34 & 138.85 \\
\hline & $f_{7}$ & $f_{8}$ & $f_{9}$ & $f_{10}$ & $f_{11}$ & $f_{12}$ \\
\hline$f_{e}$ & 153.74 & 159.36 & 182.97 & 187.66 & 200.42 & 215.39 \\
\hline$(\mathrm{n}, \mathrm{m})$ & $(0,1)$ & $(6,1)$ & $(3,2)$ & $(7,1)$ & $(1,2)$ & $(8,1)$ \\
\hline$f_{h}$ & 153.74 & 159.36 & 182.97 & 187.66 & 200.42 & 215.39 \\
\hline
\end{tabular}

Influence of the CSF compressibility: As previously, we investigated the influence of the sound celerity $c_{0}$ on the natural frequencies. Analytical and numerical solutions are reported in Table 6 . Natural frequencies do not significantly vary even within a 
Table 6. Natural frequencies vs. Compressibility

\begin{tabular}{|c|c|c|c|c|c|c|}
\hline & $f_{1}$ & $f_{2}$ & $f_{3}$ & $f_{4}$ & $f_{5}$ & $f_{6}$ \\
\hline$c_{0}=1000 \mathrm{~m} / \mathrm{s}$ & 38.67 & 69.88 & 90.69 & 100.51 & 130.34 & 138.85 \\
\hline$c_{0}=500 \mathrm{~m} / \mathrm{s}$ & 38.66 & 69.87 & 90.69 & 100.51 & 130.34 & 138.85 \\
\hline$c_{0}=300 \mathrm{~m} / \mathrm{s}$ & 38.65 & 69.85 & 90.68 & 100.47 & 130.29 & 138.84 \\
\hline
\end{tabular}

quite large band $c_{0} \in[300,1450] \mathrm{m} / \mathrm{s}$, showing that the fluid-structure interaction problem may be based on the CSF incompressibility. The pressure problem simplifies into $\Delta p=0$.

Influence of the CSF thickness: We investigated the influence of the CSF thickness on natural frequencies. To this purpose, we take for the brain radius $R_{2}=0.090 \mathrm{~m}$ and we vary $e:=R_{1}-R_{2}$. Analytical and numerical results are reported in Table 7 showing that natural frequencies increase with the CSF thickness. When the skull was assumed elastic but fixed at its external boundary, we found the same natural frequencies and modal shapes as for the rigid skull.

Table 7. Natural frequencies vs. CSF thickness

\begin{tabular}{|c|c|c|c|c|c|c|}
\hline & $f_{1}$ & $f_{2}$ & $f_{3}$ & $f_{4}$ & $f_{5}$ & $f_{6}$ \\
\hline$e=0.012 m$ & 34.24 & 62.94 & 87.19 & 91.82 & 120.42 & 133.35 \\
\hline$e=0.009 m$ & 29.42 & 55.07 & 81.64 & 83.96 & 108.57 & 128.23 \\
\hline$e=0.006 m$ & 23.87 & 45.57 & 68.82 & 80.96 & 93.07 & 117.89 \\
\hline$e=0.003 m$ & 16.80 & 32.75 & 50.51 & 69.70 & 78.17 & 89.99 \\
\hline
\end{tabular}

\section{Elastic-acoustic-elastic model (free skull)}

A more realistic problem would let the boundary $\Gamma_{0}$ of the skull free. The free vibrations of the brain-CSF-skull system are governed by [1] with conditions:

$$
\left\{\begin{array}{ccccc}
\sigma\left(\mathbf{u}_{i}\right) \mathbf{n} & = & -p \mathbf{n} & \left(\Gamma_{i}\right) & \\
\frac{\partial p}{\partial n} & = & \rho_{0} \omega^{2} \mathbf{u}_{i} \cdot \mathbf{n} & \left(\Gamma_{i}\right) & i=1,2 \\
\sigma\left(\mathbf{u}_{2}\right) \mathbf{n} & = & 0 & & \left(\Gamma_{0}\right)
\end{array}\right.
$$

Analytical solutions: Using the same technique as previously, introduction of the analytical solutions of [1] into the boundary and transpiration condition [6] leads to a dispersion equation (El-Baroudi, 2007):

$$
H_{\text {eae-free }}(\omega, n)=0
$$

Natural frequencies are thus solutions of this equation. Maple software was used to derive and solve it. Numerical solutions: Numerical solutions of [6] are obtained 
with finite element method using the variational equation, which takes exactly the same form as for the fixed skull. Only the space of test function is different $\mathbf{v}_{2} \neq 0$ on $\Gamma_{0} . \forall(\mathbf{v}, \phi) \in V \times Q$ in which $V=H^{1}\left(\Omega_{1}\right) \times H^{1}$ et $Q=H^{1}(\Omega)$. The same discrete system is also obtained. An appropriate descending analysis technique was used in the Comsol software for avoiding numerical spurious pressure modes. Analytical solutions was obtained by Maple software. The first 10 natural frequencies are reported in Table $8, f_{i}=\omega_{i} / 2 \pi$ (frequencies larger than $200 H z$ are not reported).

Table 8. Natural frequencies : Analytical vs. Numerical

\begin{tabular}{|c|c|c|c|c|c|c|}
\hline & $f_{1}$ & $f_{2}$ & $f_{3}$ & $f_{4}$ & $f_{5}$ & $f_{6}$ \\
\hline$f_{e}$ & 37,78 & 69,63 & 90,72 & 100,42 & 130,30 & 137,67 \\
\hline$(\mathrm{n}, \mathrm{m})$ & $(2,1)$ & $(3,1)$ & $(1,1)$ & $(4,1)$ & $(5,1)$ & $(2,2)$ \\
\hline$f_{h}$ & 37,78 & 69,63 & 90,72 & 100,42 & 130,30 & 137,67 \\
\hline & $f_{7}$ & $f_{8}$ & $f_{9}$ & $f_{10}$ & $f_{11}$ & $f_{12}$ \\
\hline$f_{e}$ & 153,74 & 159,34 & 182,71 & 187,66 & & \\
\hline$(\mathrm{n}, \mathrm{m})$ & $(0,1)$ & $(6,1)$ & $(3,2)$ & $(7,1)$ & & \\
\hline$f_{h}$ & 153,74 & 159,34 & 182,71 & 187,66 & & \\
\hline
\end{tabular}

Influence of the CSF compressibility: The influence of the sound velocity $c_{0}$ on the natural frequencies is obtained in Table 9. Frequencies are not influenced by the sound velocity for $c_{0} \in[300,1450] \mathrm{m} / \mathrm{s}$. This again means that in the present fluid-structure interaction we can assume incompressibility. The same simplification as previously holds for the acoustical problem.

Table 9. Natural frequencies vs. CSF compressibility

\begin{tabular}{|c|c|c|c|c|c|c|}
\hline$f_{i}(\mathrm{~Hz})$ & $f_{1}$ & $f_{2}$ & $f_{3}$ & $f_{4}$ & $f_{5}$ & $f_{6}$ \\
\hline$c_{0}=1000$ & 37,78 & 69,63 & 90,72 & 100,42 & 130,30 & 137,67 \\
\hline$c_{0}=500$ & 37,78 & 69,63 & 90,72 & 100,42 & 130,30 & 137,67 \\
\hline$c_{0}=300$ & 37,77 & 69,61 & 90,71 & 100,38 & 130,25 & 137,66 \\
\hline
\end{tabular}

Influence of the CSF thickness: Let us now consider the influence of the CSF thickness $e$ on the natural frequencies. To that purpose, we took the inner radius $R_{2}$ of $\Omega_{2}$ to $0.090 \mathrm{~m}$ and we varied the radius $R_{1}$ of $\Omega_{1}$. The natural frequencies are strongly dependent on the CSF thickness. 
Table 10. Natural frequencies vs. CSF thickness

\begin{tabular}{|c|c|c|c|c|c|c|}
\hline$f_{i}(\mathrm{~Hz})$ & $f_{1}$ & $f_{2}$ & $f_{3}$ & $f_{4}$ & $f_{5}$ & $f_{6}$ \\
\hline$R_{1}=0,078$ & 33,39 & 62,67 & 87,23 & 91,71 & 120,37 & 132,20 \\
\hline$R_{1}=0,081$ & 28,63 & 54,78 & 81,52 & 84,00 & 108,51 & 127,12 \\
\hline$R_{1}=0,084$ & 23,17 & 45,28 & 68,68 & 81,01 & 92.99 & 117,85 \\
\hline$R_{1}=0,087$ & 16,27 & 32,51 & 50,38 & 69,61 & 78,21 & 89,94 \\
\hline
\end{tabular}

Modal shapes: $\quad$ Three modal shapes are reported in Figure 2. The von Mises stress field is represented since it plays a key role for understanding either the deep brain injury at low frequency loading. Modal shapes illustrate that the highest strain

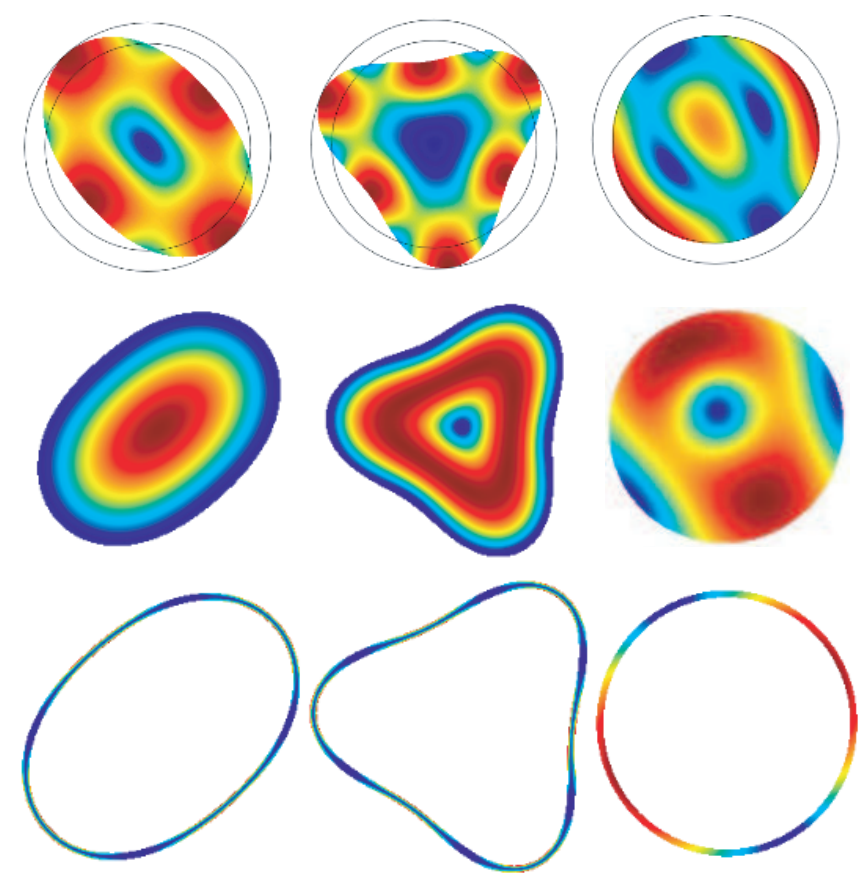

Figure 2. First three modal shapes of the brain-CSF-skull system (von Mises stress). (a) First row for elastic-acoustic model (rigid skull) : brain modal shapes; (b) Second and third rows for elastic-acoustic-elastic model (free): brain modal shapes and skull modal shapes (Highest value in red and blue for lowest stress)

occur in the central region when accounting for the fluid-interaction. This is not the case when the skull is assumed rigid. It seems to suggest that traumatic brain injury at low frequency is localized at the central region and results into a diffuse axonal injury. 


\section{Concluding remarks}

The knowledge of the natural frequencies may be very important either in understanding Trauma Brain Injury during impacted head (Willinger et al., 1996); or in the controlling the mechanical wave propagation within the brain tissue during activities (Kao et al., 2007) or neuroimaging (Hiltunen et al., 2006), (Will et al., 2007). Very simplified models have been developed in the present study for analyzing the influence of the cerebrospinal fluid phase on the frequency spectrum on the brain-CSF-skull system. Some concluding remarks could be drawn :

- it seems that the incompressibility condition is justified by simulating the sound velocity $c_{0}$ of the fluid. CSF compressibility might be neglected during the modal analysis. Indeed, within the interval $c_{0} \in[300,1450] \mathrm{m} / \mathrm{s}$ of the sound velocity, an incompressibility assumption can be used for fluid-structure in the acoustical approximation. However, compressibility could not be neglected in a dynamical simulation as Trauma Brain Injury,

- modal shapes of the rigid skull are the same as those of the elastic skull case if the outer skull is fixed. This is probably due to the high stiffness of the skull compared to that of the brain. In such a case, it is therefore possible to replace the "stiff skull" by a infinitely rigid wall. But in all cases, it seems not a very realistic model for analyzing the head impact,

- there is a significant difference between the modal shapes of rigid skull model and that of elastic skull model free at the outer boundary. This highlights the necessity of accounting as accurately as possible not only the fluid-structure interaction but also the deformability of element during a stress analysis of the impacted head,

- it is shown that the CSF thickness has a strong influence on the natural frequencies. Nevertheless, results seem to be in contradiction with the intuition. Indeed, increasing the fluid thickness tends to decrease the frequencies since the added mass increases accordingly. This apparent contradiction can be explained with the greater contribution of the skull stiffness compared to the added mass of the CSF,

- it is clearly stated that analytical and numerical results are in good agreement in this study. By the way, this validates the use of Comsol Multiphysics software for further dynamical analysis of the brain-CSF-skull system. Indeed, care should be taken when using finite element models for fluid-structure interaction simulation since non-symmetrical system may induce spurious modes (Bermúdez et al., 1995).

Currently, head numerical models contain detailed geometrical description of anatomical components inside the head by using three-dimensional imaging techniques. However, lack of accurate descriptions of non-linear brain material behaviour and interfaces (CSF) inside the head remain serious limitations for model reliability. For analyzing wave propagation within the brain tissue, it is not obvious to which extent numerical artefacts influence the overall responses. It is very difficult to distinguish the sources of discrepancies between theoretical model and experiment results, namely mathematical model assumptions and the numerical solution procedure artefacts. The two-dimensional and linear constitutive laws assumption certainly present 
a drastic limitation of the present model. Simulation of dynamical impact of the head in three-dimensional configuration is ongoing in our laboratory.

\section{References}

Bermúdez A., Durán R., Muschieti M. A., Rodrigez R., Solomin J., “ Finite element vibration analysis of fluid-solid systems without spurious modes", SIAM Journal of Numerical Analysis, vol. 32, p. 1280-1295, 1995.

Charalambopoulos A., Dassios G., Fotiadis D., Massalas C., "Frequency Spectrum of the Human Head-Neck System”, International Journal of Engineering Science, vol. 35, $\mathrm{n}^{\circ} 8$, p. 753-768, 1997.

El-Baroudi A., Analyse modale paramétrique en interaction fluide-structure. Applications aux problèmes issus de la biomécanique, Rapport de recherche, Institut de Recherche Mathématique de Rennes, Sep., 2007.

Hault-Dubrulle A., Contribution à l'amélioration des connaissances des phénomènes d'interaction fluide-structure à l'intérieur de la boîte crânienne soumise à une sollicitation dynamique : études expérimentales et numériques, Thèse de doctorat, Université de Valenciennes et du Hainaut Cambrésis, 2007.

Hiltunen J., Hari R., Jousmaki V., Mueller K., “ Quantification of mechanical vibration during Diffusion Tensor Imaging at 3 T”, NeuroImage, vol. 32, p. 93-103, 2006.

Kao Y.-H., Guo W.-Y., J.-K. L. A., Hsiao Y.-H., Chou C.-C., “ The Respiratory Modulation of Intracranial Cerebrospinal Fluid Pulsation observed on Dynamic Echo Planar Images", Magnetic Resonance Imaging, vol. 26, n² 2, p. 198-205, 2007.

Kleiven S., Finite Element Modeling of the Human Head, Thèse de doctorat, Royal Institute of Technology, Stockholm, Sweden, 2002.

Morand H. J. P., Ohayon R., " Substructure variational analysis of the vibrations of coupled fluid-structure systems. Finite element results", International Journal of Numerical Methods and Engineering, vol. 14, p. 741-755, 1979.

Rakotomanana R., Eléments de dynamique des solides et structures déformables, Presses Polytechniques et Universitaires Romandes, Lausanne, To appear 2008.

Will U., Berg E., “ Brain Wave Synchronization and Entrainment to Periodic Acoustic Stimuli”, Neuroscience Letters, vol. 424, p. 55-60, 2007.

Willinger T., Taleb L., Kopp C., " Modal and temporal analysis of head mathematical models”, Traumatic brain injury: bioscience and mechanics, Mary Ann Liebert Inc., Larchmont, p. 265-276, 1996. 